\title{
SPEX: THE SPECTROPOLARIMETER FOR PLANETARY EXPLORATION
}

\author{
J.H.H. Rietjens ${ }^{1}$, F. Snik ${ }^{2}$, D.M. Stam ${ }^{1}$, J.M. Smit ${ }^{1}$, G. van Harten $^{2}$, C.U. Keller ${ }^{2}$, A.L. Verlaan ${ }^{3}$, E.C. Laan ${ }^{3}$, R. \\ ter Horst ${ }^{4}$, R. Navarro ${ }^{4}$, K. Wielinga ${ }^{5}$, S.G. Moon ${ }^{6}$, R. Voors ${ }^{7}$ \\ ${ }^{I}$ SRON Netherlands Institute for Space Research, Sorbonnelaan 2, 3584 CA Utrecht, the Netherlands \\ ${ }^{2}$ Sterrekundig Instituut Utrecht, Princetonplein 5, 3584 CC Utrecht, the Netherlands \\ ${ }^{3}$ TNO Science \& Industry, Stieltjesweg 1, 2628 CK Delft, the Netherlands \\ ${ }^{4}$ NOVA-ASTRON, Oude Hoogeveensedijk 4, 7991 PD Dwingeloo, the Netherlands \\ ${ }^{5}$ Mecon Engineering BV, Koopmanslaan 25, 7005 BK Doetinchem, the Netherlands \\ ${ }^{6}$ Cosine, Niels Bohrweg 11, 2333 CA Leiden, the Netherlands \\ ${ }^{7}$ Dutch Space, Newtonweg 1, 2333 CP Leiden, the Netherland
}

\begin{abstract}
We present SPEX, the Spectropolarimeter for Planetary Exploration, which is a compact, robust and low-mass spectropolarimeter designed to operate from an orbiting or in situ platform. Its purpose is to simultaneously measure the radiance and the state (degree and angle) of linear polarization of sunlight that has been scattered in a planetary atmosphere and/or reflected by a planetary surface with high accuracy. The degree of linear polarization is extremely sensitive to the microphysical properties of atmospheric or surface particles (such as size, shape, and composition), and to the vertical distribution of atmospheric particles, such as cloud top altitudes. Measurements as those performed by SPEX are therefore crucial and often the only tool for disentangling the many parameters that describe planetary atmospheres and surfaces. SPEX uses a novel, passive method for its radiance and polarization observations that is based on a carefully selected combination of polarization optics. This method, called spectral modulation, is the modulation of the radiance spectrum in both amplitude and phase by the degree and angle of linear polarization, respectively. The polarization optics consists of an achromatic quarter-wave retarder, an athermal multiple-order retarder, and a polarizing beam splitter. We will show first results obtained with the recently developed prototype of the SPEX instrument, and present a performance analysis based on a dedicated vector radiative transport model together with a recently developed SPEX instrument simulator.
\end{abstract}

\section{INTRODUCTION}

Spectropolarimetry is a technique in which not only a flux spectrum from a particular source is measured, but also the state of polarization at each wavelength. This technique is particularly useful for studying planetary atmospheres, since sunlight, scattered in a planetary atmosphere and/or reflected by a planetary surface contains a wealth of information on the scattering and reflecting particles comprising the planetary atmosphere or surfaces [1]. Knowledge of microphysical properties of the scattering particles, such as size, shape and composition is essential for understanding how planetary atmospheres work.

Because of the complex and often time-dependent composition of the planetary atmospheres, retrieving the physical properties from observed flux spectra is often not accurate enough or leads to degeneracies. However, it has been shown that spectral knowledge of the degree of polarization can vastly improve the retrieval, since the polarization signal is much more sensitive to the microphysical properties of the scattering particles than the flux signal [2]. The degree of polarization of reflected sunlight is generally very low at small phase angles. For an outer planet, such as e.g. Jupiter, polarimetry is thus most advantageous with a polarimeter on a spacecraft that passes the planet in a fly-by or one that orbits the planet.

In the past, there have been several spacecrafts that carried instruments with polarimetric capabilities, such as Pioneers 10 and 11, Voyager 1 and 2, the Galileo mission, and both the Saturn orbiter and the Huygens probe of the Cassini mission. The polarimetry in these instruments was/is based on multiple flux measurements through broadband polarisation filters in two or three orientations. This has the disadvantage that the separate flux measurements are not performed simultaneously, and usually through different optical systems (e.g. different polarization filters). As a result, the degree of polarization that is obtained can have systematic errors of several percent, which is too large to use polarimetry to its full potential. In addition, the degree of linear polarization shows at least as much spectral features as the flux [3], which are not resolved by the broadband measurements.

The SPEX instrument (Spectropolarimeter for Planetary EXploration) [4],[5], uses a novel polarimetric technique to simultaneously measure the flux, the degree and the direction of linear polarization of scattered and reflected sunlight from 400 to $750 \mathrm{~nm}$, with the spectral resolution of the measured flux spectra of about $2 \mathrm{~nm}$, and that of the polarization spectra somewhat larger.

In this paper we describe the conceptual, optical and mechanical design of the SPEX instrument. Also, we present performance simulations for SPEX on one of the spacecraft of the Europa Jupiter System Mission 
(EJSM). Finally, we discuss the solar system bodies for which SPEX is perfectly suited to measure the atmospheric and surface properties.

\section{INSTRUMENT CONCEPT}

The Spectropolarimeter for Planetary EXploration (SPEX) is an innovative, compact instrument. The spectropolarimetry is achieved by encoding the degree of linear polarization (DoLP) and angle of linear polarization (AoLP) of the incident light in the measured flux spectra using the technique of spectral modulation [6]. The spectral modulation principle allows the polarization optics to be very small and robust. The spectrally modulated spectrum is measured using a spectrometer and detector yielding sufficient spectral resolution. SPEX has the capability to measure spectra under different viewing angles while flying over a ground pixel. This way SPEX samples the flux and polarization scattering phase function over a large range of scattering angles, yielding a precise characterization of the atmospheric aerosols. Limb viewers can be employed for the study of high clouds and vertical profile information on aerosols.

In contrast to the polarimeters on previous missions, spectral modulation [6],[7] allows the flux and polarization spectrum to be obtained with a single measurement. This greatly reduces the susceptibility to differential effects related to different optical and detector properties, and temporal effects such as changing scenes or seeing while observing in orbit. With spectral modulation the full linear polarization properties of the light are encoded into a sinusoidal variation of the flux spectrum such that the amplitude of the sinusoid scales with DoLP and its phase scales with AoLP. The spectral modulator can be constructed from standard, passive optical components: a combination of an achromatic quarter-wave retarder, an athermal multiple-order retarder and a polarizer. With appropriate orientation of the polarization optics [6], any spectrum $\mathrm{s}_{0}(\lambda)$ is spectrally modulated as:

$$
S(\lambda)=\frac{1}{2} S_{0}(\lambda) \cdot\left[1 \pm \operatorname{DoLP}(\lambda) \cdot \cos \left(\frac{2 \pi \cdot \delta(\lambda, T)}{\lambda}+2 \cdot \operatorname{AoLP}(\lambda)\right)\right],
$$

with the sign depending on the orientation of the polarizer. Using a polarizing beam-splitter, the modulations for the two beams are out of phase and the flux spectrum is obtained trivially by adding the spectral measurements (after compensating for transmission differences). It was shown that this spectral modulation furnishes linear spectropolarimetry with relatively large accuracy and sensitivity [6].

Typical specifications of the SPEX instrument are listed in Table 1. The current instrument prototyping activities are based on and tailored to a mission including a Martian orbiter. Due to its novel design, SPEX is a compact instrument, with low volume, low mass, and low power budget.

\section{DESIGN}

The current design of SPEX is tailored to a Mars orbiter at an altitude of 300-400 km. As shown in Fig. 1, the multi-viewing angle capability of SPEX is implemented by incorporating nine fixed fields of view along the flight direction, each equipped with the necessary polarization optics. The two outermost viewing apertures have a rotated field of view in order to function as limb viewers.

\section{A. Optical design}

The optical system of SPEX can be divided into two main parts: the pre-slit polarization encoding optics (see Fig. 2a), and the spectrometer optics (see Fig. 2b). The nine fields of view, of which each individual field is split according to two orthogonal polarization states, are imaged within the sides of the $12.5 \times 12.5 \mathrm{~mm}$ detector.

Table 1: Specification of SPEX

\begin{tabular}{|l|l|}
\hline spectropolarimeter volume & $\sim 1$ liter \\
\hline mass including electronics & $\sim 2 \mathrm{~kg}$ \\
\hline maximum power consumption & $\sim 2 \mathrm{~W}$ \\
\hline spectral range & $400-750 \mathrm{~nm}$ \\
\hline spectral resolution for polarization & $20 \mathrm{~nm}$ \\
\hline spectral resolution for flux & $2 \mathrm{~nm}$ \\
\hline viewing directions & $7+2$ limb viewers \\
\hline field of view for each viewing direction & $1^{\circ} \times 7^{\circ}$ (cross-flight, to deal with planet's rotation) \\
\hline pupil size for each viewing direction & $1.1 \mathrm{~mm}^{2}$ \\
\hline measured polarization properties & Stokes I, Q \& U \\
\hline polarization sensitivity & 0.005 (degree of linear polarization) \\
\hline relative polarization accuracy & $5 \%$ (down to 0.01 absolute) \\
\hline
\end{tabular}




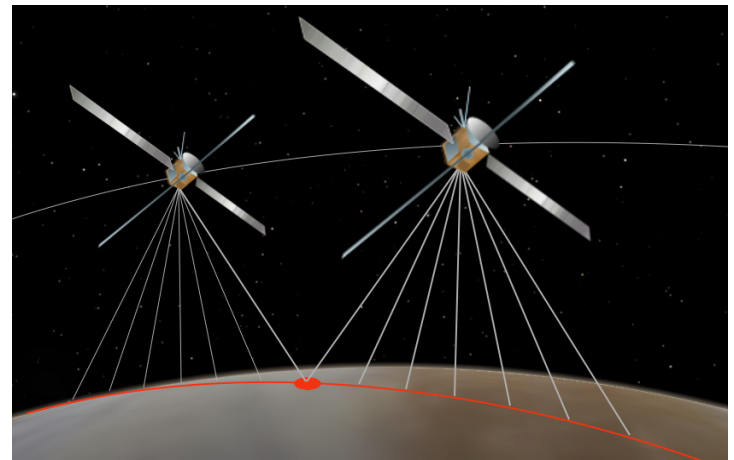

Fig. 1 Impression of SPEX on an orbiting platform. Each ground pixel is viewed from all 7 downward viewing apertures. The limb viewers are not shown in this image.

The incoming beam, which has a diameter of $1.1 \mathrm{~mm}$, will first pass the polarization optics to ensure zero instrumental polarization: a BK7G18 Fresnel rhomb for the achromatic quarter-wave retarder, an athermal combination of sapphire and $\mathrm{MgF}_{2}$ for the thick retarder and a Wollaston prism for the polarizing beam-splitter, see Fig. 2a. The radiation tolerant BK7G18 is chosen for the Fresnel rhomb because this optical element receives the highest radiation flux. The combination of sapphire and $\mathrm{MgF}_{2}$ retarder plates with the correct thickness ratio ensures good athermal behavior, which is necessary since the measurement of the AoLP is very sensitive to changes in the total retardance. The dispersion within the Wollaston prism (out of $\alpha-\mathrm{BBO}$ ) is largely compensated by the dispersion caused by the off-axis light path through the lenslets. Only one single lenslet $(\mathrm{F}=10 \mathrm{~mm})$ focuses the two polarized beams on two separated slits. All optical components are non-moving.

The light from all nine apertures is focused onto a common slit plane, which in turn is imaged via a transmission grating and spectrometer optics onto a focal plane array. Light enters the slit with a focal ratio of $\mathrm{F} / 10$, and is rescaled by the camera lens into $\mathrm{F} / 3.3$ in order to have a good match with the detector and the specified spectral resolution. The current design has been optimized for an altitude of $300 \mathrm{~km}$, with viewing angles of $0, \pm 14, \pm 28, \pm 42$, and \pm 56 degrees. The imaging optics contains three radiation resistant glass lenses of which two are identical aspheres (see Fig. 2b). For reasons of compactness a (quartz) transmission grating is used, having a spacing of 750 lines per mm. Spectral overlap of orders is partially solved by the near zero quantum efficiency of the intended detector outside $400-1000 \mathrm{~nm}$, which means that together with the low transmission of the radiation resistant F2G12 lens at shorter wavelengths, the 350-400 nm regions will not be registered. Further filtering of the unwanted wavelengths $(750-1000 \mathrm{~nm})$ is done by using a blocking filter. At least $80 \%$ of the energy of all fields and wavelengths should be focused within an area of $2 \times 2$ pixels, $(50 \times 50$ $\mu \mathrm{m})$, which has been achieved with this optical design.

\section{B. Mechanical design}

Several renderings of the mechanical design of the SPEX prototype are shown in Fig. 3. The SPEX prototype is designed for environmental testing of the instrument to allow for TRL6 qualification. The design is very stiff, yet light $(0.9 \mathrm{~kg})$ and compact ( $<1$ liter $)$. The main frame is made out of a solid block of $\mathrm{Al}$ of $12 \times 12 \times 6 \mathrm{~cm}$ into a structure of $\sim 1.5 \mathrm{~mm}$ thickness. Modern production techniques like spark eroding and diamond turning allowed designing a prototype that requires no active alignment of optical elements, other than the focusing of the spectrometer detector.

(a)

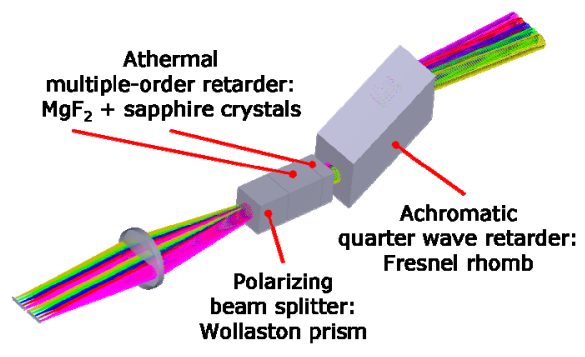

(b)

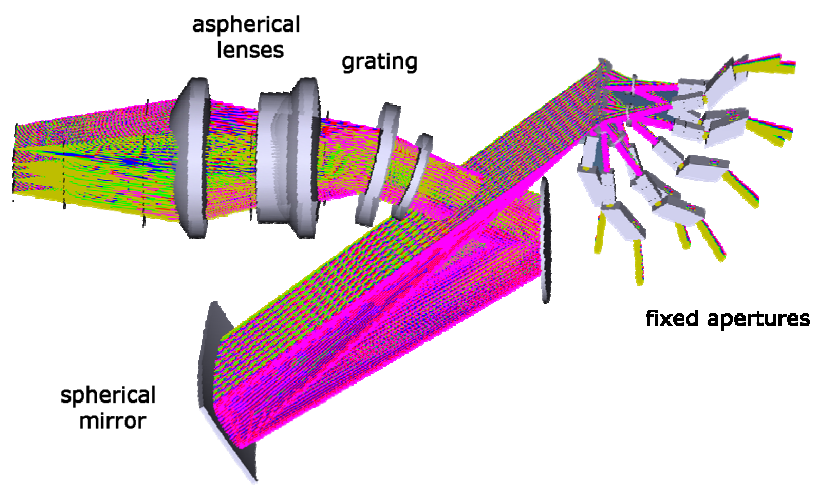

Fig. 2 Optical design of SPEX: a) pre-slit polarization encoding optics. b) pre-slit and spectrometer optics. 


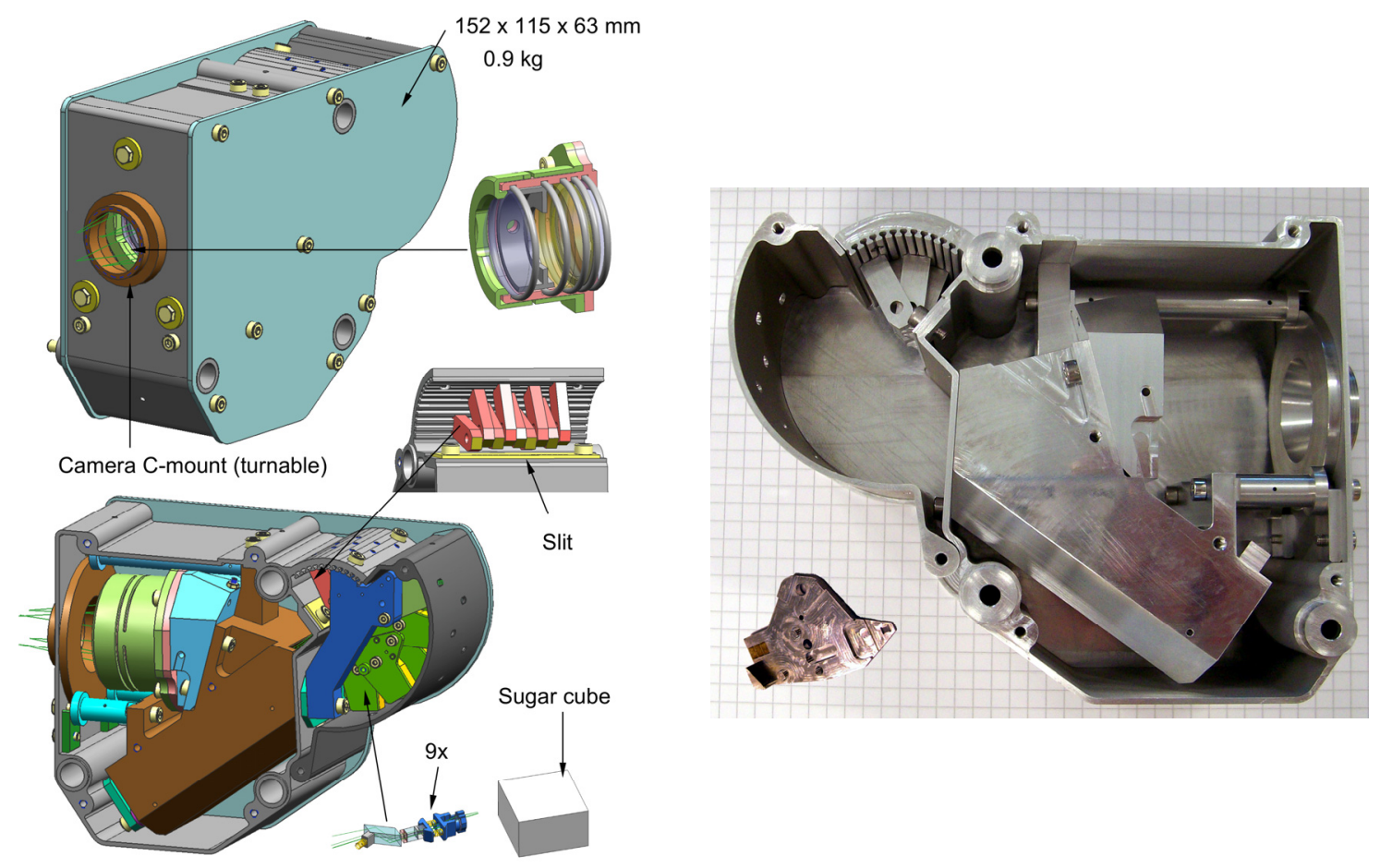

Fig. 3 Mechanical design (left) of SPEX showing the exterior, interior, and zooms of the imaging optics, beam combiner, and polarization pre-optics. Photograph (right) of the partly assembled housing of the SPEX prototype. A part of the polarizing pre-optics housing is shown separately.

A number of numerical simulations have been performed to verify and support the mechanical design. This comprises Finite Element Model (FEM) thermal analysis, stiffness and strength calculations. In particular a large effort was made to reduce the stresses exerted on the elements of the polarization optics. In general changes of stress on optical elements will result in variation of the birefringence. As a result the polarization measurement would be affected in an unpredictable manner and after the calibration of the instrument. A solution is to mount the optical elements very stiff to survive the launch vibrations, but yet with very low forces to minimize the induced stresses.

\section{SPEX PERFORMANCE}

The performance of the SPEX instrument is analyzed with a dedicated end-to-end simulator [5]. In short, the simulator uses as input representative Stokes-vector spectra for each viewing aperture that is simulated. These input spectra are calculated with a polarized radiative transfer code that is based on an adding-doubling algorithm [8]. The effect of each (optical) component of SPEX on the flux and polarization state of the Stokesvector spectrum is modeled using appropriate Mueller matrices for each component. The simulated signal measured by the focal plane array is obtained from the flux component of the Stokes vector using a binning operation. Detector properties are modeled using global parameters based on manufacturer specification sheets. A demodulation technique is used in order to transform the signal from the focal plane array into spectra of the DoLP and AoLP [6]. The simulator is easily adapted to investigate alternative designs of SPEX, e.g. with different spectral resolution and modulation period, and a different set of viewing apertures.

Here we show a full simulation for the case of SPEX observing Jupiter's equator from a large distance, corresponding to the mission profile of the EJSM. The planetary phase angle is $60^{\circ}$. In this case, the SPEX instrument is simulated with 7 viewing apertures looking at longitudes $15^{\circ}$ apart (no limb viewers), a spectral range of $480-840 \mathrm{~nm}$, and increased retardance of the multiple order retarder yielding higher resolution of the measured polarization signal. The simulated (raw) output of the focal plane array for a cloudy atmosphere without haze layer is shown in Fig. 4a. Image brightness corresponds to the simulated flux of the signal. For reference, the spectral range corresponding to the horizontal direction of the focal plane array is indicated. The simulated image shows several aspects of typical SPEX measurements.

- The spectra from the separate viewing apertures can be clearly distinguished.

- Spectra from each viewing aperture consist of two groups corresponding to the two output beams from the polarizing beam splitter, the bottom one is $s$-polarized, the top one is $p$-polarized. 
(a)
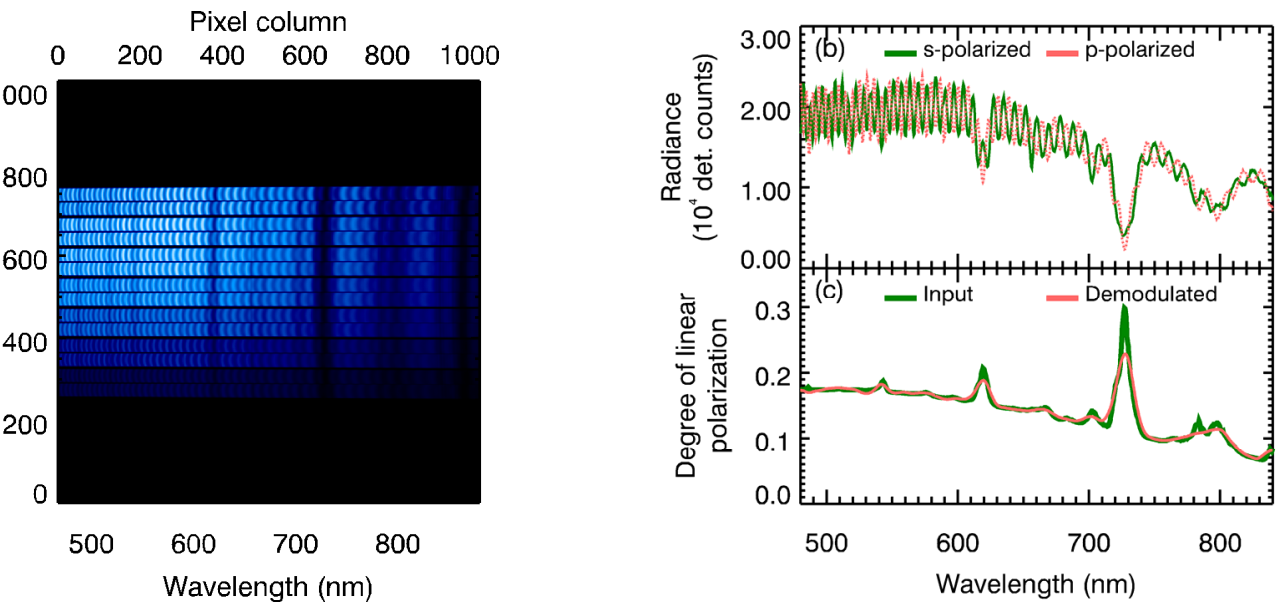

Fig. 4 a) Simulated image the focal plane array of a Jupiter observation, with a cloudy atmosphere and a planetary phase angle of $60^{\circ} . \mathrm{b}$ ) $s$ - and $p$-polarized spectrum from viewing aperture 3. c) Input and demodulated DoLP from viewing aperture 3 .

- Spectra from different viewing apertures show a different flux and modulation amplitude, depending on the local illumination and viewing geometries on the planet.

- The field-of-view behavior of the multiple-order retarder manifests itself in curved maxima and minima over the swath range of the s- and p-polarized output beams.

From Fig. 4a we have extracted the $s$ - and $p$-polarized spectra from a single aperture, which are plotted in Fig. $4 \mathrm{~b}$ for further analysis. The spectra clearly show several methane absorption bands, the $180^{\circ}$ phase difference between the $s$ - and $p$-polarized spectra, and the spectrally varying modulation amplitude that is indicative of a spectrally dependent DoLP. More details of the spectra are described elsewhere [9]. Using a demodulation algorithm, the DoLP and AoLP can be obtained from the $s$ - and p-polarized spectrum. Both the input and demodulated DoLP are shown in Fig. 2c. The demodulated DoLP is in very good agreement with the input DoLP, indicating the high accuracy that can be achieved using the spectral modulation principle. This is vital for discriminating between different atmospheric scenes. Fig. 5a shows the calculated flux spectra for a cloudy Jupiter atmosphere, and a cloudy atmosphere with a haze layer on top. Hardly any difference between the two flux spectra is visible, illustrating the need for spectropolarimetry. The differences in the incident and demodulated DoLP of the reflected light for the two atmospheres are shown in Fig. $5 \mathrm{~b}$ and Fig. 5c respectively. Clearly, the contrast between the two atmospheres is much larger in the DoLP spectra. And equally important, SPEX is able to measure these differences with high accuracy, allowing discriminating between the two atmospheres and identifying their properties. The demodulated DoLP also partially resolves the methane features in the spectra, which contain additional information about the aerosol properties and their vertical distribution [8].

\section{OUTLOOK}

SPEX has the potential to contribute significantly to space missions aiming at investigating planetary atmospheres and surfaces including Earth from an orbiting platform. Examples are the Europa Jupiter System Mission (as shown above), the Titan Saturn System Mission, the Lunar Lander, a future Mars orbiter, and installation on the International Space Station. Below, we summarize the main objects of interest for SPEX and the science that SPEX can deliver.
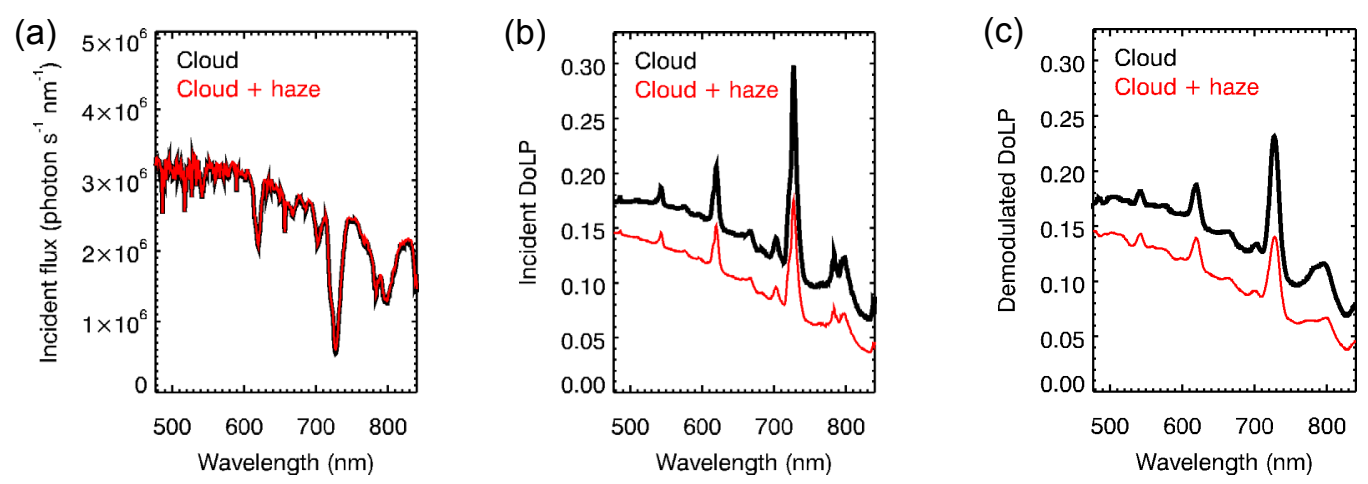

Fig. 5 a) Calculated flux for two Jupiter atmospheres. b) Calculated DoLP for two Jupiter atmospheres for viewing aperture 3. c) Demodulated DoLP corresponding to the input DoLP of b). 
On Earth, the contribution of aerosol and cloud particles to the radiative balance and hence the climate has been recognized as an extremely important one, while detailed knowledge on the essential properties of these particles (size, shape, composition, distribution) is still lacking. Here, SPEX could play a key role.

From the Moon, SPEX could observe the whole Earth during all seasons and through all phases, just as if it were an exoplanet. Such SPEX measurements would help tremendously the design of spectropolarimeters for exoplanetary research, and the development of retrieval algorithms, because polarimetry is considered to be a powerful tool for detecting and characterizing exoplanets [9]. Spectropolarimetric observations of Earth could serve as a benchmark for the interpretation of future spectropolarimetric observations of Earth-like exoplanets.

The main science goal for SPEX on Mars would be the characterisation of the distribution and microphysical properties of dust particles in the atmosphere. Since the gaseous atmosphere of Mars is very thin, atmospheric dust has a strong influence on the atmosphere's radiative balance. In addition, dust particles play an important role in heterogeneous chemistry: its surfaces facilitate reactions and thus act as sources and/or sinks of trace gases like the biomarker methane. The limb viewers of SPEX allow the study of $\mathrm{H}_{2} \mathrm{O}$ or $\mathrm{CO}_{2}$ ice crystals that form thin clouds high in the atmosphere, which is essential for understanding the water cycle, the dynamical processes high in the atmosphere, and their impact on the Martian climate.

In orbit around Jupiter, SPEX can characterize the composition, size and shape of the cloud and haze particles, which would help to explain the colors of features like the bands and ovals, and the structure of the atmosphere (cloud and haze altitudes and vertical extension). Such information is required for understanding the radiative and chemical balances, and hence the dynamical processes in the atmosphere. Understanding Jupiter's atmosphere will help to better understand the formation and evolution of planets. In orbit around the moons Europa and Ganymede, which are thought to have a subsurface ocean below an icy crust, SPEX can study their surface composition and roughness. The roughness of the icy surface could reveal locations where subsurface water wells up from below; a key towards establishing the existence of the ocean, the thickness of the ice layer, and the chances of life forms below the ice. Also, the composition of Europa's ice layer could reveal the presence of organic materials, while the composition and roughness of Ganymedes surface would provide knowledge on the weathering processes that take place and on the evolution of the moon.

On a spacecraft orbiting Titan, SPEX can characterize the upper haze layer of Titans atmosphere, and it could be used to observe Saturn and its rings. A more interesting place for SPEX would be on a balloon. From there, SPEX could observe sunlight that has been diffusely transmitted through Titans atmosphere, and characterize the haze and cloud particles and their temporal and spatial variations. From below the balloon, SPEX could also observe the surface, which is important for understanding the observations of scattered sunlight, and for getting more information on the sources and sinks of Titans methane cycle, which are thought to be found on and in the surface.

\section{ACKNOWLEDGMENTS}

The design study for SPEX is supported by a PEP grant from NIVR (Nederlands Instituut voor Vliegtuigontwikkeling en Ruimtevaart; Netherlands Agency for Aerospace Programmes).

\section{REFERENCES}

[1] J.E. Hansen, L.D. Travis, "Light scattering in planetary atmospheres”, Space Scie. Revs., Vol. 16, pp. $527-$ 610 (1974)

[2] J.E. Hansen, J.W. Hovenier, "Interpretation of the polarization of Venus", J. Atmospheric Sci., Vol. 31, pp. $1137-1160$ (1974)

[3] I. Aben, F. Helderman, D.M. Stam, and P. Stammes, "Spectral fine-structure in the polarization of skylight”, Geophys. Res. Lett., Vol. 26, pp. 591-594 (1999)

[4] F. Snik, et al, "SPEX: an in-orbit spectropolarimeter for planetary exploration", Proc. SPIE 7010, p. 701015 (2008)

[5] F. Snik, et al, "SPEX: The Spectropolarimeter for Planetary EXploration", Proc. SPIE 7731, p. 773147 (2008)

[6] F. Snik, T. Karalidi, and C.U. Keller, "Spectral modulation for full linear polarimetry", Applied Optics, Vol. 48 Issue 7, pp.1337-1346 (2009)

[7] K. Oka, and T. Kato, 'Spectroscopic polarimetry with a channeled spectrum', Optics Letters, Vol. 24, pp. 1475-1477 (1999)

[8] J. De Haan, P. Bosma, J. Hovenier, "The adding method for multiple scattering calculations of polarized light”, Astron. Astrophys., 183, 371-391 (1987).

[9] D. M. Stam, J. W. Hovenier, and L. B. F. M. Waters, "Using polarimetry to detect and characterize Jupiterlike extrasolar planets", $A \& A 428,663-672$ (2004)

[10] D. M. Stam and J. W. Hovenier, "Errors in calculated planetary phase functions and albedos due to neglecting polarization", $A \& A 444,275-286$ (2005) 